\title{
Carnets
}

Revue électronique d'études françaises de l'APEF

Première Série - 2 Numéro Spécial | 2010

Littératures nationales: suite ou fin. Résistances, mutations \& lignes de fuite

\section{Rearticuler histoire(s) et littérature}

\section{Marc Quaghebeur}

\section{(2) OpenEdition}

12 Journals

\section{Édition électronique}

URL : http://journals.openedition.org/carnets/5322

DOI : $10.4000 /$ carnets.5322

ISSN : 1646-7698

Éditeur

APEF

Édition imprimée

Date de publication : 1 juin 2010

Pagination : 255-270

Référence électronique

Marc Quaghebeur, "Rearticuler histoire(s) et littérature », Carnets [En ligne], Première Série - 2 Numéro Spécial | 2010, mis en ligne le 16 juin 2018, consulté le 24 septembre 2020. URL : http:// journals.openedition.org/carnets/5322 ; DOI : https://doi.org/10.4000/carnets.5322

\section{(c) (i) (9)}

Carnets est mis à disposition selon les termes de la licence Creative Commons - Atribution - Pas d'utilisation commerciale 4.0 International. 
REARTICULER HISTOIRE(S) ET LITTERATURE

MARC QUAGHEBEUR

Archives et Musée de la Littérature

Bruxelles 
Parler de littérature nationale, c'est tout d'abord interroger la notion de "nation" qui la sous-tend. Si cette dernière l'engendre pour une large part, c'est à la littérature nationale qu'il est souvent demandé de conforter la notion de nation.

$\mathrm{Au}$ sens où nous l'entendons aujourd'hui, cette notion est relativement récente. Du moins si nous nous penchons sur le temps long de l'histoire humaine. Elle entre par exemple en contradiction avec les notions d'empire. Paradoxe toutefois: certaines des nations européennes les plus emblématiques n'arriveront à leur réalité nationale qu'à travers des formes d'expansions impériales, coloniales.

C'est clairement, à travers l'Histoire de France et les formes de son unification, que la notion de nation prend la plupart des dimensions qui sont aujourd'hui encore les siennes. Et c'est très précisément au XVIII ${ }^{\mathrm{e}}$ siècle - et notoirement à travers la Révolution française que le terme acquiert le sens que nous lui connaissons. La nation, on le sait, s'y substitue au roi. N'est-ce pas au cri de "Vive la nation!" que les troupes de la Révolution française s'élancent à Valmy contre celles du roi de Prusse.

La préhistoire de l'engendrement du concept n'est point sans intérêt. D'autant que, comme il est logique au vu des spécificités de l'unification française, le fondement conceptuel du terme ne concerne pas uniquement la citoyenneté, qui paraît aujourd'hui lui être consubstantielle - ce que la Révolution, tout aussi logiquement, imposa. Cette perception occulte toutefois une part de ce qui s'est joué dans la reformulation moderne de ce vocable.

La question de l'unification linguistique du pays autour du français, langue de la Cour et de Paris, est en effet essentielle à son histoire. Entre la fin du XVII et le milieu du XVIII ${ }^{\mathrm{e}}$ siècle, l'Académie française, que le cardinal de Richelieu mit sur pied dans la cohérence d'un grand dessein politique, lie progressivement la notion de nation à la communauté de langage. À l'époque, pourtant, la plus grande partie du domaine royal français n'est pas de langue maternelle française. L'abbé Grégoire ne manquera pas de le rappeler, quelques décennies plus tard, dans son plaidoyer pour l'éradication des autres langues du pays comme de ses patois: ses chiffres indiquent une proportion d'un cinquième de la population qui parlerait français. Sous la Restauration, on s'approcherait de la moitié.

À travers la dynamique propre au XVIII siècle, puis avec l'accélération des romantismes nationaux qui font suite à l'épopée napoléonienne, la fusion entre communauté de langue, appartenance et fidélité à un État territorial et politique dessinent progressivement les bases de ce que va devenir pour beaucoup la citoyenneté nationale. Cela s'engendre et s'actualise, je le rappelle, en France, pays européen alors au faîte de son apogée. Pour des raisons que j'ai exposées ailleurs, langue et littérature sont clairement inscrites dans ce pays, dès le XVII ${ }^{e}$ siècle, au centre du dispositif d'unification et d'identité des populations qui le constituent. Les $\mathrm{XIX}^{\mathrm{e}}$ et $\mathrm{XX} \mathrm{X}^{\mathrm{e}}$ siècles pousseront ce processus à son terme. 
À la même époque, on parle également en Europe de nation allemande alors que les populations qui parlent et écrivent cette langue sont réparties sur de nombreux États, regroupés pour l'essentiel au sein du Saint-Empire romain de la nation germanique. De cette réalité historique et humaine bien spécifique, différente de celle qui prévaut en France mais dynamisée par la violence des invasions napoléoniennes, surgit le concept de langue comme Volksgeist. II se répand en Europe et débouche, par exemple, au XIX ${ }^{\mathrm{e}}$ siècle, dans la partie de la Belgique à dominante néerlandophone sur la formule; "de taal is gans een volk"1. Quoique différent - il se situe en amont -, le Volksgeist n'est pas contradictoire du concept français de nation mais provient - et entend exprimer, à la même époque - l'évidence d'une vaste communauté linguistique éparpillée entre divers principats. La nation à la française provient, elle, d'un pays plurilingue que ses élites entendent rendre plus cohérent par la pratique de leur langue.

Rien de contradictoire en revanche entre les deux acceptions pour ce que le mot induit du rapport langue-littérature. Autre chose est de la citoyenneté, les deux guerres mondiales se chargeront de le rappeler. La France ne réunit-elle pas dans son imaginaire et sa réalité des hommes que n'assemble pas a priori une communauté pouvant se fonder sur la sensation d'appartenance immémoriale à une même langue?

L'Europe produit donc une symbiose progressive entre territoire, État, langue et Littérature. Le XIX ${ }^{e}$ siècle en constitue le siècle d'or, avec ses luttes de libération nationale et la nécessité, pour chacune de celles-ci, de se fonder sur une identité, à la fois prospective et rétrospective. Ces identités trouvent leurs repères dans une vision mythologique de l'Histoire d'une part; dans un corpus littéraire directement articulé à celle-ci, de l'autre.

L'extension et la spécification du concept se produisent dans ce creuset du XIX ${ }^{e}$ siècle qu'est le Traité de Vienne (1815). Les réactions qu'il suscite très rapidement vont en effet dans un tout autre sens que celui souhaité par les puissances signataires et avivent les idées de citoyenneté et de liberté qui ont accompagné la spécification du mot en France. Elles s'imbriquent en outre dans la seconde vague d'expansion coloniale européenne. Celleci sera le fait de la Grande-Bretagne et de la France, puis de la Belgique; et, dans une moindre mesure, de l'Allemagne et de l'Italie. Cette considération est plus importante qu'il n'y paraît. Les processus d'expansion des trois pays colonisateurs, comme ce fut d'ailleurs le cas jadis pour l'Espagne, le Portugal puis les Pays-Bas, permirent en effet un devenir de ces États et de leurs habitants qui créa ou renforça une conscience nationale. D'autant mieux que les contradictions inhérentes au concept de nation - en tous les cas dans sa dimension identitaire liée à la langue - pouvaient être subsumées au travers de l'expansionnisme du pays.

\footnotetext{
${ }^{1}$ La langue est tout un peuple.
} 
Il est donc logique que le ressac de la notion de nation se produise notamment après les Indépendances. Cela fait émerger, de façon tensionnelle ou imprévue, des complexités humaines qui étaient déjà le propre des sociétés concernées mais que, relié comme il l'était aux expansions coloniales, le concept de nation avait permis de nier ou de contenir. Ce ressac découle aussi des deux guerres civiles européennes du $X X^{e}$ siècle, prétendument mondiales qui opposèrent, jusqu'à la folie et l'abjection, des populations auprès desquelles l'appartenance et l'identité nationales avaient été exacerbées en dogme foncièrement négateur de l'Autre. Le conflit de 1914-1918 et les Traités de Versailles et de Trianon les avaient amenées à une exacerbation plus grande encore.

L'ambigüité - pour ne pas dire les impasses - de la construction européenne de la fin du $X X^{e}$ siècle est loin d'avoir produit en outre ce qu'une invention de réalité(s) politique(s) européenne(s) différente de celle dans laquelle nous sommes plongés eût permis d'engranger. Le dépassement positif du concept de nation et des entités et populations qu'il avait réunies à un moment du développement historique européen (pour leur permettre de répondre notamment aux nécessités du marché liées aux manufactures et à l'industrie lourde) paraît de jour en jour plus menacé ou improbable. La réalité européenne que nous connaissons aujourd'hui contribue toutefois aussi à la déstabilisation relative du vocable; à sa perte de pertinence dynamique. Sans profiler pour autant une identité européenne, mais en réactivant des micro-nationalismes dans lesquels l'extrême-droite fait son lit, et où s'enlisent des formations politiques dont le travail idéologique ne s'est jamais penché sérieusement sur les soubassements du concept de nation.

Impossible qu'une telle situation soit sans effet sur le concept et la réalité des littératures nationales! Et d'autant plus que le concept est directement lié à l' "invention" des nations européennes, mais aussi (désormais) à celles des pays qu'ont produits ou repropulsés les processus coloniaux. Si cette invention européenne ne fut possible, à maints égards, qu'à travers le développement parallèle des expansions coloniales ${ }^{2}$, les "nations" de ce que l'on appelait le Tiers Monde ne pourront que remettre en cause certains fondements du concept de littérature nationale puisque la quasi-totalité de ces pays sont plurilingues. Si l'on veut comprendre certaines questions qui se posent aujourd'hui à nous, il faut à la fois prendre en compte la question des rapports entre nation et expansion au détriment de tiers, mais aussi ce que l'Hégire à l'européenne amène en retour.

\footnotetext{
${ }^{2}$ Dans d'autres pays -, à commencer par l'Allemagne - les volontés d'expansion en Europe au détriment d'autrui et/ou d'extermination de "boucs émissaires" ne sont vraisemblablement pas sans liens avec une situation globale de développements nationaux qui connut des cours différents, mais pas vraiment contradictoires, dans les divers pays.
} 
Profondément marqués par le modèle intrinsèque foncier de la notion de la nation, désormais confrontée à elle seule après les ressacs ${ }^{3}$ de la fin des empires coloniaux, les débats qui sont les nôtres dévoilent enfin à l'œil nu ce qui noue le concept. Cela nous contraint à nous interroger sur le visage masqué qui est souvent celui de l'Histoire; comme sur les conséquences, différentes certes, qui sont et seront celles d'un tel schéma dans les divers pays passés par les tutelles coloniales européennes. À l'heure de la mondialisation néo-capitaliste et de la mise en œuvre sur le "vieux Continent" de structures de fonctionnement insuffisantes pour dépasser le cadre des nations qui ont fait l'Europe, n'y aurait-il pas lieu d'aller au fond des non-dits et de ce que furent les conditions de possibilités de l'idée de nation?

À ces considérations générales s'ajoute bien incidemment le fait, pour la recherche, de la nécessité d'examiner concrètement chaque cas particulier. De voir - donc - comment s'est préparée et réalisée l'idée nationale dans des contextes aussi différents que ceux de l'Espagne et du Portugal, de l'Italie et de l'Allemagne, de la France et de la GrandeBretagne, de la Pologne et de la Russie, des pays nordiques ou balkaniques.

Là où des nations (c'est le cas du Portugal) correspondent réellement, et de longue date, à une entité linguistique, la réalité n'est pas la même que dans des pays, telle la France, où cette réalité est le fruit d'une politique pluriséculaire. Encore moins dans des pays comme la Belgique ou la Suisse dans lesquels ce type d'unification n'a jamais pu voir le jour, mais qui n'en constituent pas moins des réalités à part entière - le noyau fascisant du concept d'État-nation entendît-il les réduire à l'inexistence.

Le concept de littérature nationale a correspondu à des nécessités. II s'est produit et fut proféré dans des contextes précis. Chaque fois, il s'agissait d'arriver à la construction de ces États-nation qui ont fait l'Europe des derniers siècles. Pour y arriver, rien de tel que la projection et la diffusion d'un ensemble de textes susceptibles de dépasser, et les clivages politiques internes, et la complexité des réalités historiques de l'autre. II s'agissait en somme de mettre les citoyens en rapport avec une forme d'imaginaire dont les peuples ont toujours eu besoin. Les traditions orales, les légendes et les épopées y avaient répondu jadis. Elles n'étaient plus pertinentes pour le projet national. Elles devinrent donc folklore.

Par rapport à la question des littératures nationales, la construction française a joué, une fois encore, un rôle décisif. Elle est, on ne le rappellera jamais assez, le fruit d'un très long processus. Ne procède-t-elle pas d'une volonté constante des différentes élites qui se

\footnotetext{
${ }^{3}$ Les diverses gesticulations de l'actuel président de la République française, Nicolas Sarkozy (discours de Dakar; débat sur l'identité nationale; expulsion des Roms) ne sont bien évidemment pas étrangères à de tels ressacs même si elles ne caractérisent pas toute la société française.
} 
sont succédé - et parfois opposé - à la tête de la France? Ce pays, l'un des plus centralisés du monde, assigne à la langue française un rôle qui figure dans sa constitution, rôle qui résulte de maints dispositifs pratiques et symboliques pluriséculaires destinés à unifier territoires et populations plurilingues à l'origine. Ce travail de symbolisation s'est préparé avant même que n'émerge l'idée moderne de nation, autour et à partir de la littérature. Au long des siècles - la dynamique des Lumières et de la Révolution française y contribuant décisivement, je l'ai dit plus haut -, la littérature fut vécue et présentée comme une sorte de transcendance de la langue; de point d'identification (au-delà des différences) des Français; et de valeur majeure de leur image à l'étranger - jusqu'à Nicolas Sarkozy du moins ${ }^{4}$.

Le choix de ce type de point nodal du dispositif identitaire est logique dès lors que les instances de la France décidèrent progressivement d'unifier le pays et de le symboliser autour de la langue des élites. Au moment où l'idée de nation prend sérieusement forme et s'apprête à se répandre dans l'Europe du XIX ${ }^{e}$ siècle - dans la foulée entre autres du remueménage créé par les armées de l'Empire français -, la littérature est devenue l'équivalent presque parfait de la civilisation et du bon goût français. Pour la plupart des peuples européens et pour les Français eux-mêmes, elle peut dès lors prendre figure de lieu majeur de projection mythique des réalités nationales en cours de construction. Rien d'étonnant donc à ce que, dans les pays d'Europe centrale et orientale par exemple, brassés par la logique impériale de la maison d'Autriche ou enserrés dans celle de la Sublime Porte, se soient développés des Musées de la littérature ${ }^{5}$ qui rebrassent le corpus littéraire dans une perspective pluriséculaire foncièrement mythique.

À la façon dont Gustave Lanson avait œuvré pour sa part dans sa monumentale histoire de la littérature française? Ce rapprochement, qui surprendra, est peut-être moins provocateur qu'il n'y paraît.

Au cœur du dispositif "littératures nationales", on trouve toujours un noyau dur selon lequel, à un territoire et à un peuple, correspondent une langue et une littérature, qui en est l'âme. Que se passe-t-il dès lors que l'on se trouve, non seulement à l'intérieur d'empires plurilingues (dont surgirent des écrivains majeurs), mais dans des pays de taille réduite qui sont plurilingues depuis très longtemps, et d'où naquirent aussi de grands écrivains qu'il n'est pas forcément nécessaire d'annexer au corpus des écrivains pratiquant la même langue dans un grand pays voisin? II en va ainsi de la Belgique ou de la Suisse, mais aujourd'hui aussi de l'Autriche.

Dans les corpus belge ou suisse, on constate que, dès le début du long $\mathrm{XIX}$ siècle i. e. après les effets de la défaite de Napoléon à Waterloo - se pose la question de savoir

\footnotetext{
${ }^{4}$ Mais Dominique de Villepin, à droite, ou Laurent Fabius, à gauche, contredisent explicitement cette rupture avec une tradition essentielle. Ils n'auraient jamais imaginé de lancer un débat sur l'identité française puisqu'ils l'incarnent et la creusent notamment dans leurs textes.

${ }^{5}$ Le Musée de la littérature qui s'ouvrira à Vienne en 2013 relaiera ce type de réalités.
} 
comment inventer une littérature nationale dans un État où cohabitent plusieurs langues. Voire, de savoir si l'on a le droit, dans ces pays, d'en avoir une dès lors que la réalité politicoétatique dans laquelle se trouvent les citoyens ne correspond à aucune langue propre?

Les effets à long terme des concepts de nation et de littérature nationale n'ont cessé de produire des effets d'ordres divers, et jusqu'à aujourd'hui. On les interroge insuffisamment. Pourquoi le $\mathrm{II}^{\mathrm{e}}$ Reich, par exemple, a-t-il pu fantasmer et faire fantasmer autour de revendications au terme desquelles tout qui parle ou écrit allemand devait faire partie d'un seul et même pays? L'Anschluss et la question des Sudètes, prélude du démembrement de la Tchécoslovaquie, en ont procédé. L'on sait d'autre part le rôle que la littérature a joué - et joue encore - dans certains processus de revendication "nationale" de populations inscrites dans des pays plurilingues (Catalans ou Basques en Espagne, Flamands en Belgique, etc.).

Pour ce qui est de la France elle-même, et en dépit de la question de l'universel qui fait aussi partie (depuis la fin du XVIII ${ }^{\mathrm{e}}$ siècle, en tous les cas) du noyau fantasmatique de son identité nationale, c'est à partir de ces équivalences - tout sauf évidentes - que découle la difficulté, voire l'impossibilité, de la prise en compte sérieuse dans l'Hexagone des réalités des littératures francophones. Tel n'est pas (ou n'est plus) le cas des domaines hispanophone, lusophone ou anglophone.

L'incapacité de certains responsables français d'accepter que figurent, aux frontières de la République, et en tant que réalités autonomes, des entités où se trouvent des francophones, procède du même noyau fantasmatico-idéologique. Les propos récurrents de Jean-Pierre Chevènement sur la Belgique sont en la matière particulièrement significatifs mais s'inscrivent dans une tradition fort ancienne qui visa par exemple le Grand-Duché de Luxembourg en 1919. La volonté, chez nombre de commentateurs, de réduire le mot "francophone" aux anciens sujets de la France est tout aussi révélatrice d'une situation et d'une pensée dans laquelle il n'est pas question d'assumer le pluriel qui présida à l'invention de cette langue.

Non contente d'être née dans des contextes précis de formation des États-nations, la notion de littérature nationale véhicule en outre des reliquats fantasmatiques de réalités que ces littératures auraient voulu produire, mais qui n'ont pas foncièrement ou totalement abouti. Prendre en considération l'Histoire au sein de chacun de nos pays, et le faire en relation avec la littérature (faire de même pour ce qui est de la Littérature et de l'Histoire) a toutes chances, il est vrai, d'amener à des perceptions beaucoup plus subtiles que celles des dénégations ontologisantes des littératures nationales. L'approche des littératures francophones fait donc courir le risque d'en apprendre plus sur les littératures et sur leur lien avec les réalités historiques que les doxa pétrifiées des littératures nationales. 
Le concept de littérature nationale, qui a certes permis de mobiliser des foules de lecteurs à certains moments de nos Histoires, pose aujourd'hui question. Et cela, tant par rapport à la transformation de nos champs de vie qu'à l'égard de l'approche du fait littéraire en tant que tel. Or nos manuels demeurent profondément marqués par ces visions mythologiques.

En réaction se sont mises en place des formes d'approche nouvelles, souvent fractionnées et très partielles, qui s'inaugurent dans les années 1960 par la querelle Barthes/Picard. Elle eut le mérite de dénoncer les simplifications de I'histoire positiviste par rapport à ce qui se joue dans l'écrit littéraire. Elle n'a pas débouché pour autant sur une refondation du rapport Histoire/Littérature, enlisé dans l'amidon des littératures nationales (entre mythe et positivisme) mais sur des savoirs parcellaires qui risquent de contribuer à la négation progressive du fait littéraire comme à l'étude de son ancrage dans l'Histoire laquelle n'est pas un contexte mais un creuset. Si la notion de littérature nationale doit être profondément remise en cause, il ne s'agit donc pas pour autant d'abandonner l'approche historique des textes ni d'occulter le rapport de ceux-ci avec les entités nationales à l'intérieur desquelles ils ont été produits, ou dont ils proviennent. II s'agit en revanche de sortir de l'équivalence quasi automatique instaurée par des traditions exégétiques pour les raisons que j'ai esquissées plus haut.

Une telle approche, qui ne peut se limiter à l'espace "national" de l'écrivain, doit par exemple être au moins croisée avec le dynamisme général de l'espace linguistique auquel appartient cet écrivain. Et, par ailleurs, avec le dynamisme, tout aussi profond mais différent (puisque provenant souvent de traductions), des courants régionaux, continentaux ou mondiaux qui contribuent non seulement à la circulation des idées mais aussi à l'invention de formes nouvelles. Les cas des écrivains changeant de langue, comme celui des écrivains migrants posent par ailleurs des questions qu'il s'impose d'insérer dans la problématisation historique dont je parle. Elles ne se résolvent pas à travers le fractionnement des études, dont les Gender Studies, les Postcolonial Studies, etc. sont un symptôme.

Il est temps de multiplier les travaux comparatifs et de le faire notamment au niveau intralinguistique, n'en déplaise aux tenants de la vieille discipline comparatiste. Ainsi pourrat-on arriver à mieux comprendre, et les processus historiques généraux, et les spécifications nationales.

Prenons comme exemple la question du symbolisme. Ce phénomène culturel a marqué globalement l'espace européen, de l'Europe du Sud à l'Europe du Nord et jusqu'à la Russie. II y a donc là une fabuleuse matière de circulation des formes et des influences dans un continent, à laquelle d'aucuns ont d'ailleurs consacré de beaux travaux. Pour ce 
mouvement comme pour d'autres (naturalisme, surréalisme, etc.), il y aurait toutefois intérêt à le faire non seulement à travers les espaces translinguistiques mais aussi à travers les espaces de la même langue. C'est ce à quoi s'est par exemple attachée Maria Jesus de Cabral à partir de la question de l'héritage mallarméen ${ }^{6}$.

Mallarmé avait longuement réfléchi aux modalités d'un théâtre nouveau mais ne l'avait pas écrit. En revanche - et son article après la première de Pelléas et Mélisande de Maeterlinck demeure, aujourd'hui encore, une des lectures les plus subtiles de cette pièce c'est un Belge, Maurice Maeterlinck, qui va réaliser ce théâtre. II faudrait encore aller plus loin que les commentaires de Maria de Jesus Cabral, et analyser ce qui se passe au même moment chez Claudel $^{7}$, dramaturge majeur dont l'œuvre ne sera reçue que dans un effet différé. Celui-ci ne tient pas uniquement à l'éloignement extrême-oriental du diplomate. S'il y eut d'autre part quelques traces actives de réception du symbolisme en Suisse - mais seulement autour de Genève -, on ne peut pas dire que ce mouvement ait joué un rôle important dans le devenir des lettres romandes au sein de la Confédération helvétique alors qu'en Belgique I'héritage des pré-symbolistes français (Baudelaire, Rimbaud, Mallarmé, Verlaine) produisit des effets majeurs, non seulement dans la génération fin de siècle, mais bien au-delà. Seule, l'Histoire peut expliquer des situations aussi différentes entre trois pays proches.

N'est-ce pas à partir de tels phénomènes qu'il conviendrait d'enfin commencer à travailler afin de mieux déterminer ce que les réalités nationales induisent à l'intérieur des champs littéraires? L'impact du "national" dans le champ littéraire s'en trouvera éclairé sans pour autant être le vecteur de sauts interprétatifs mythologisants. Ce sera en outre une belle façon de remettre la littérature à une juste place, dans l'enseignement comme dans l'ordre des connaissances. Par ce biais, l'on accédera beaucoup plus, et beaucoup mieux, à la complexité des Histoires européennes ou africaines, comme à la complexité de la fabrication littéraire.

La mythologie qui servit d'englobant au déploiement des littératures nationales permit à nos ascendants récents de disposer de beaux contes d'enfants. Ceux-ci, même s'ils ont toujours cours, ne correspondent plus aux réalités auxquelles sont confrontés nos descendants. II est donc plus qu'urgent de reprendre à frais nouveaux la question de l'articulation de la Littérature et de l'Histoire. Et de le faire sans continuer à fantasmer à partir de noyaux imaginaires, devenus obsolètes à maints égards, que la pérennité relative des États européens contribue à entretenir.

\footnotetext{
${ }^{6}$ Maria de Jesus Cabral, Mallarmé hors frontières. Des défis de l'œuvre au filon symbolique du premier théâtre maeterlinckien, Préface de F. Schuerewegen, Amsterdam/New York, Éditions Rodopi, 2007, (collection. "Faux Titre "), $362 \mathrm{p}$.

${ }^{7}$ L'un a lu La Princesse Maleine; l'autre, La Jeune Fille Violaine.
} 
Cette perspective à laquelle j'invite me paraît d'autant plus nécessaire que l'on se trouve, depuis les Indépendances africaines, devant des réalités littéraires qui ne peuvent se subsumer sous le concept sricto sensu de littérature nationale - celui de l'équivalence langue / territoire. Les pays d'Afrique libérés de la tutelle coloniale produisent des textes dans les grandes langues de leur colonisation (anglais, français, portugais), mais aussi dans les langues vernaculaires - et elles sont parfois très nombreuses à l'intérieur d'un même pays. Pour les pays du Maghreb par exemple, vieux pays de l'écrit, on trouve évidemment, outre le français, l'arabe classique, mais aussi le berbère et les variations dites dialectales de l'arabe.

Chacun de ces nouveaux champs littéraires nationaux, articulé d'autre part à des réalités et réseaux transnationaux, a bien évidemment à voir avec l'Histoire du pays. C'est ce qu'il s'agirait de montrer, dans le respect des complexités et des pluralités linguistiques - et non dans les simplifications liées à l'hypostase fallacieusement univoque de la langue. Cela continue toutefois de déranger, particulièrement dans l'aire francophone, et ce n'est pas un hasard. Le phénomène est toutefois bien plus large. Pourquoi n'y a-t-il toujours pas en Belgique, par exemple, d'histoire comparée des corpus littéraires francophone et néerlandophone? Pourquoi ne dispose-t-on pas d'études de ce qui les amarre chacun à l'histoire nationale et régionale d'une part; aux champs linguistiques transnationaux des deux langues concernées de l'autre; aux interférences, intérieures et extérieures, enfin? C'est évidemment le cœur même des idéologies mortifères et claustrales qui serait atteint. Clercs et politiques n'ont donc aucun intérêt à interroger les bases de leurs fonds de commerce.

Longtemps, en effet, il s'est agi de construire, à travers la littérature, des identités. On a pourtant pu voir, et à plusieurs reprises, sur le continent européen, les effets ravageurs de ces modules. Pourquoi persister dans l'absurde sinon parce qu'un lien social s'y est tissé, et que celui qui pourrait le remplacer se trouve encore dans les limbes? L'un des paradoxes du concept de littérature nationale, n'est-il pas qu'à travers l'étude de ce qui tire par excellence vers le complexe, l'on ait pu être amené, au travers des discours d'escorte nationalistes, à des simplifications souvent grossières? Ce phénomène montre bien comment l'idée mythique de l'Histoire qui domina les $X I X^{e}$ et $X X^{e}$ siècles, d'une part empêcha de faire l'Histoire, et, de l'autre, produisit des réalités culturelles et scientifiques dont il nous faut aujourd'hui sortir de toute urgence.

Pour les chercheurs que nous sommes, il s'agit de comprendre et de faire comprendre ce qui se passe dans l'écriture - notamment à travers son articulation à l'Histoire. II s'agit donc de comprendre également ce qui peut se produire de différent au travers d'une même langue.

Que la remise en cause du noyau idéologique du concept de littérature nationale mine certaines assises de l'imperium littéraire français ne fait bien évidemment aucun doute 
mais n'a rien de périlleux pour la France et les Français - l'obsolescence d'un mythe desservant in fine ceux qui s'y accrochent. II donne au contraire une chance nouvelle à notre langue. L'examen attentif de nombreux champs littéraires ne manque d'ailleurs pas d'amener à de telles remises en cause. On peut augurer en outre que les réalités contemporaines y contraindront de plus en plus.

Qu'un écrivain francophone comme Édouard Glissant ait proposé la notion d'archipélagie, au-delà et à travers le combat postcolonial qui est le sien, n'est bien évidemment pas un hasard. Cela devrait amener tout participant aux espaces de la langue française à y réfléchir. II ne s'agit pas pour autant d'entrer dans une dérive générale visant, comme certains le voudraient, la déperdition de l'objet. II s'agit, au contraire, d'aboutir à la conception, plus concrète, d'un objet dans sa complexité.

Un tel projet va susciter, longtemps encore, de solides résistances, particulièrement dans le champ qui est le mien: l'espace de la langue française et des littératures francofrancophones. J'ai rappelé tout à l'heure les résistances auxquelles ces littératures continuent de devoir faire face. En témoigne, entre autres choses, la place extrêmement congrue qu'elles occupent par exemple dans l'espace académique de la République française.

Cette place congrue - et incongrue - est toutefois aussi le fait des pays francophones, même s'ils ont inscrit peu ou prou un corpus francophone dans les marges de leurs programmes. Pour autant, ils n'ont pas pris en main sérieusement ces corpus et leurs enjeux. Ils n'ont donc toujours pas repensé les programmes - ils se sont contentés en somme de développer quelque peu l'enseignement de la littérature francophone du cru (avec quelques ouvertures sur telle ou telle autre littérature francophone). L'on continue donc, partout, de reproduire - avec quelques aménagements - la doxa issue de la promotion de la littérature française, littérature nationale qui se répandit d'autant mieux à l'heure de l'hégémonie française qu'elle se présentait sous le couvert de l'universel.

L'ouverture timide des portes du cursus scolaire aux productions francophones de chaque pays concerné constitue certes un pas, totalement insuffisant. Seules, des refontes partielles du parcours franco-français (avec des variantes régionales, vu la diversité des mondes francophones) et des mises en perspective conjointes et comparées des littératures francophones peuvent amener à un réel redéploiement de ces espaces linguistiques et littéraires.

Ce qui s'est passé en France, l'année que le président Jacques Chirac avait décidé, à l'heure de l'achèvement de ses mandats, de consacrer aux Francophonies donne à 
penser. C'est à ce moment précis que Le Monde publie un manifeste intitulé Pour une Littérature-Monde. Ce manifeste qui voit le jour le 16 mars 2007 en page 2 du Monde des livres, est signé par quarante-quatre écrivains. Il est présenté par le quotidien français comme le manifeste "de quarante-quatre écrivains en faveur d'une langue française qui serait "intérieure", libérée enfin de son pacte exclusif avec la nation". Ce manifeste précède la publication chez Gallimard d'un ouvrage dû à Jean Rouaud et Michel Le Bris, l'instigateur du manifeste: Pour une Littérature-Monde.

Si le texte s'inscrit bien évidemment dans les perspectives de mondialisation contemporaine, il entre aussi dans un jeu de comparaison, explicitement énoncée d'ailleurs, avec l'espace et le dynamisme des littératures de langue anglaise. II se positionne d'autre part, et comme il sied, à l'égard d'une série de positions dominantes du champ francofrançais mais aussi à l'égard de la critique anglo-saxonne. On rappellera utilement en effet que certains de leurs médias avaient à plusieurs reprises annoncé à grand bruit le déclin littéraire de la France.

Le Manifeste écrit ainsi: "Fallait-il tenir pour acquis quelques dégénérescences congénitales des héritiers de l'empire colonial français en comparaison de ceux de l'empire britannique? Ou bien reconnaître que le problème tenait au milieu littéraire lui-même, à son étrange art poétique tournant comme un derviche tourneur sur lui-même, et à cette vision d'une francophonie sur laquelle une France mère des arts, des armes et des lois continuait de dispenser ses lumières en bienfaitrice universelle, soucieuse d'apporter la civilisation aux peuples vivant dans les ténèbres".

Voilà qui montre clairement, me semble-t-il, l'ancrage des rédacteurs du Manifeste dans l'Histoire impériale et post-impériale française. Leur texte comporte donc un salutaire aspect de remise en cause d'une certaine enclôture sur lui-même du milieu littéraire parisien - et ce, depuis plusieurs décennies. II témoigne tout autant de l'ambiguïté qui accompagne nombre de manifestations francophones officielles.

En revanche, le Manifeste ne remet nullement en cause la concentration lutétiotropiste du système éditorial - littéraire et symbolique - de langue française. Or, cette situation ne connaît aucun équivalent au monde. Elle n'est notamment pas le fait du monde anglo-saxon par rapport auquel le Manifeste entend se positionner. Signé par des écrivains d'origines diverses mais qui participent tous au système littéraire parisien - et y sont généralement ancrés -, le Manifeste vise en fait à renforcer le primat, voire le monopole, de la capitale française en matière littéraire à l'heure où le devenir des littératures francophones pourrait le remettre progressivement en cause.

Or, cette situation, résultat d'une Histoire, et d'une Histoire strictement nationale qui s'est proférée et drapée dans les pourpres de l'Universel, est une des causes de la stagnation relative des mondes littéraires de langue française. Fruit d'un processus 
pluriséculaire, qui s'affirme ouvertement à partir du XVII ${ }^{\mathrm{e}}$ siècle, ce monopole n'a pas connu d'hiatus symbolique fondamental, malgré l'extension de l'empire français et les effets de la décolonisation, ni la diversité des pays européens dans lesquels s'est inventé le français. Tout à la logique même de ce processus pluriséculaire, qu'il ne lui paraît pas devoir remettre en question, le Manifeste renforce à frais nouveaux le monopole parisien alors que celui-ci hypothèque le devenir à moyen et long terme des littératures de langue française.

Avec une rare violence et des simplismes qui font rêver, le Manifeste se dresse donc contre l'idée francophone en feignant ou en croyant respecter les littératures francophones. Le Manifeste écrit notamment que "L'émergence d'une littérature-monde en langue française consciemment affirmée, ouverte sur le monde, transnationale, signe l'acte de décès de la francophonie." À la décharge des signataires, on notera que le Manifeste pointe à sa façon la confusion créée par la France entre espaces culturels francophones et francophonie, confusion dommageable aux civilisations dans lesquelles le français constitue un des éléments de l'être-au-monde. L'instance politique chargée aujourd'hui de rassembler des pays soucieux de faire contrepoids à l'hégémonie anglo-saxonne ne porte-t-elle pas le nom de Francophonie - alors que bien des pays qui la composent n'ont, à proprement parler, rien de francophone ${ }^{8}$ ?

Cet état d'esprit désigne non seulement un dessein politique français postcolonial ou postimpérial mais dessert doublement les desseins qu'il entend défendre. Celui d'un rassemblement des peuples de langues diverses contre l'hégémonie du modèle anglosaxon, d'une part. Celui des peuples, d'autre part, au sein desquels le français est - avec d'autres langues, certes! - constitutif de l'Histoire et de l'Identité, mais qui n'ont pas forcément partie liée à l'autre enjeu. Le choix du mot Francophonie pour des réalités différentes (où ce qui est en train de l'emporter, ce ne sont pas les Francophonies historiques mais l'organisation d'États soucieux de faire contrepoids à Londres et Washington) n'est pas un hasard. II plonge dans une histoire conceptuelle où le singulier l'a toujours emporté sur le pluriel.

Ce faisant, l'on est en train de rater la véritable prise en charge des réalités francophones, et l'on condamne peut-être, à moyen terme, un système dont les potentialités sont cependant uniques. On en est arrivé ainsi à une nouvelle impasse à laquelle ont entendu répondre les auteurs du Manifeste. Hélas, sans analyse suffisante des fondements du système; et avec la prolifération de nouveaux simplismes. "Personne ne parle le francophone, ni n'écrit en francophone." écrivent les signataires.

Cette évidence - elle ne fait que reprendre de très anciens poncifs (personne ne parle le belge écrivait-on il y a un siècle) liés à une vision transcendantale de la langue -

\footnotetext{
${ }^{8}$ Certains font même partie de la lusophonie.
} 
n'est pas pertinente, sous le vernis de son apparente évidence, pour la prise en compte des diverses littératures francophones et de leurs logiques. À la fois spécifiques et tensionnelles (elles sont, et autonomes, et liées à l'espace culturel franco-français), ces littératures se trouvent dans une situation telle qu'aujourd'hui encore, la circulation entre elles est quasiment nulle si elle ne passe pas par Paris. Leurs pôles éditoriaux n'ont toujours pas décollé hors de leurs prés carrés respectifs les plus étroits.

Cela découle très évidemment de la façon dont l'histoire de France amena ses élites à s'approprier comme un bien propre une langue que deux ou trois autres pays européens avaient inventée, au même titre que les habitants du Nord de la France. Et cela, pour en faire une nation. Les coordonnées de celle-ci sont donc singulières. Elles expliquent entre autres "l'exception française" où l'identité passe largement par une identification avec une langue, qui semble dès lors avoir constitué de toute éternité un bien propre. Ce fait ne peut qu'être reprofilé si l'on veut tenir compte de ce qu'induisent les réalités francophones. Ou amener à la dénégation de ce qu'elles portent et apportent.

Que le Manifeste, fût-ce malgré lui, s'inscrive dans cette logique pluriséculaire vaut d'être pointé. II en dit long sur la pérennité des dispositifs symboliques, alors même qu'ils ne correspondent plus au réel. Ceux-ci continuent en effet de s'imposer à ceux-là même qui prétendent en sortir. Que, pour ce faire, ces derniers en arrivent de fait à dénier la pluralité des littératures francophones dès lors que leurs textes ne sont pas promus et avalisés par Paris, constitue d'une part une vieille rengaine (il n'est bon bec que de Paris, paraît-il), mais procède aussi, qu'on le veuille ou non, d'une stratégie - et d'une stratégie promotionnelle. Car les concepteurs du Manifeste savent pertinemment qu'il existe, de par le monde, des littératures de langue française. Ils écrivent d'ailleurs qu'elles forment "un vaste ensemble dont les ramifications enlacent plusieurs continents".

L'erreur - et ce n'est pas un hasard - tient évidemment, et une nouvelle fois, à la confusion, dommageable, entre francophonie et littératures francophones. J'ai plaidé de longue date pour une séparation claire des fonctions. Le combat contre l'hégémonie anglosaxonne et l'alliance avec des pays dans lesquels le français n'est pas constitutif de l'histoire propre, ne peut se faire sérieusement à l'enseigne d'un mot aussi connoté que francophonie - qui plus est, mis bien évidemment au singulier. Après les strates de la première vague coloniale européenne dans laquelle la France n'occupait pas la place centrale (Canada, Louisiane, Antilles, Sénégal, Guyane, Réunion, Maurice), les histoires coloniales, belge et française, ont amené sur la carte du monde une extension singulière du français. Celle-ci est bien différente de celle de l'anglais, de l'espagnol ou du portugais. Elle est en effet foncièrement plurielle - ce qui impose d'ailleurs l'adoption urgente du concept de francophonies, écrit comme un pluriel. 
Seules, ces francophonies ont une réalité historique et contemporaine foncière. Leur prise en compte, comme leur rayonnement, suppose le dépassement d'un système littéraire et éditorial exclusivement centré sur Paris, comme la mise en place d'instruments scientifiques et pédagogiques nouveaux d'approche des littératures. Cela suppose tout aussi évidemment un système de diffusion diversifié, adapté aux différents pays, et un dépassement de la conception obsolète de l'idée de nation. Car la situation que nous avons à affronter renvoie bien évidemment aux conséquences d'une conception de la nation qui est l'héritière directe de la construction singulière qui amena à la réalité France. Une construction qui prit environ un millénaire.

Que ce soit au moment des décolonisations que les mots "francophone" et "francophonie", qui existaient depuis près d'un siècle, aient réellement pris leur essor est logique. On ne dira toutefois jamais assez que leur sens demeure entaché des conditions de leur invention par Onésime Reclus, dans la seconde moitié du XIX siècle; et qu'il n'a pas entièrement évolué. Parce qu'il est lié à l'espace impérial français d'une part, à l'idéologie française de l'autre. Ces mots excluaient en effet les francophonies originaires que sont la Belgique et la Suisse. Ils ne valaient que pour l'empire colonial français en voie d'extension.

C'est dire comme ces termes sont doublement marqués. Et par l'histoire nationale française, et par l'histoire coloniale française. Or, faute de mots nouveaux ou de connotations nouvelles (qui ne verront pas le jour sans de profonds aggiornamento des mentalités et des structures de fonctionnement), ce passé grève une bonne part du devenir des espaces culturels de langue française - et dès lors, de la langue française elle-même. Or, ce qui est sorti de ces Histoires difficiles, et pas toujours glorieuses, ce sont précisément des littératures. Des littératures qui amènent à une remise en cause fondamentale du concept de littérature nationale tel qu'il a été produit au fil des siècles - et notamment à partir de l'histoire de France.

Cette remise en cause, qui n'est en rien la négation de l'Histoire mais son approfondissement, doit amener à un approfondissement de la perception et de l'analyse de l'historicité des réalités culturelles. Elle doit également permettre la prise en compte, dans un espace physique donné, de la cohabitation de littératures écrites en plusieurs langues. Cela forcera les chercheurs à travailler à la fois sur des histoires singulières et sur des aires transnationales, notamment liées au linguistique.

Le caractère sacré de la littérature française, entraînant de fait ou de droit sa prééminence, constitue un iceberg autour duquel n'ont cessé de tourner, même les critiques soucieux de la prise en compte de réalités littéraires de langue française en dehors de l'Hexagone. Au XIX ${ }^{\mathrm{e}}$ siècle, les Suisses Pierre-André Sayous et Virgile Rossel en donnèrent ainsi l'exemple, eux qui ne pouvaient considérer la production helvétique que comme une périphérie, une aire latérale. À partir d'une telle conception, les littératures concernées sont 
de facto dévalorisées. Un champ littéraire pluricentré et vivace devient dès lors impossible en langue française.

$\mathrm{Au} X \mathrm{XX} \mathrm{X}^{\mathrm{e}}$ siècle, les Belges jouèrent une partition en partie différente. Du moins à partir de l'émergence d'écrivains aussi singuliers que Charles De Coster, Émile Verhaeren ou Maurice Maeterlinck. Impossible de ne pas affirmer leur singularité et de la référer, en partie du moins, à l'histoire du pays. Tel sera par exemple le cas du critique François Nautet. Là encore, toutefois, les bases théoriques issues du concept d'État-nation étaient telles que les assises du discours de singularisation belge recouraient à la métaphore, voire à l'emphase lyrique.

La France elle-même se pencha sur ces curiosa venus du Nord. Elle les enroba dans le mythe nordique - invitant par ailleurs ses tenants à revenir progressivement à la norme classique. Face aux chocs de l'Histoire du $X X^{e}$ siècle, le primat symbolique et éditorial franco-français entendit très vite se réaffirmer. Les concepts de littérature française de Belgique, du Canada ou de Suisse prirent ainsi, après 1914-1918, le relais de la curieuse formule qu'utilisait par exemple Nautet, "littérature belge d'expression française", formule que l'on retrouve aujourd'hui pour maintes littératures francophones. Si la formule de Nautet renvoie à l'existence d'une littérature de langue néerlandaise dans le royaume, elle substitue à une réalité - la langue - l'emprise d'une culture - celle d'un dominant dans la langue.

Le déclin relatif des deux types de formule coïncida avec l'émergence des Francophonies et de La Francophonie institutionnelle. Cette dernière dispose de moyens financiers relativement importants. Elle suscite dès lors des productions théoriques ou des actions mais celles-ci ne contribuent pas toujours à la réelle prise en compte et à l'autonomisation, comme à la circulation, des littératures francophones. Est-ce un hasard?

C'est l'idée même de nation telle que l'Europe des trois derniers siècles l'a produite qui est remise en cause par les Francophonies. Cela se produit toutefois dans un jeu de forces périlleux, puisqu'aucune solidarité transversale suffisante n'existe à ce jour entre Francophonies (du Sud comme du Nord) et que chacun se déploie dans une belle solitude individualiste au sein d'une dynamique bilatérale dont le pôle est et demeure Paris.

C'est pourtant dans cette transformation que se joue en partie l'avenir d'une langue. Là que se trouve aussi le moyen d'habiter à frais vifs et nouveaux le concept de littérature nationale. 